\title{
L'Église et l'argent : les quêtes commandées dans le diocèse de Sherbrooke, 1893-1926
}

\section{Guy Laperrière}

\section{Volume 41, 1974}

URI : https://id.erudit.org/iderudit/1007242ar

DOI : https://doi.org/10.7202/1007242ar

Aller au sommaire du numéro

Éditeur(s)

Les Éditions Historia Ecclesiæ Catholicæ Canadensis Inc.

ISSN

0318-6172 (imprimé)

1927-7067 (numérique)

Découvrir la revue

Citer cet article

Laperrière, G. (1974). L’Église et l'argent : les quêtes commandées dans le diocèse de Sherbrooke, 1893-1926. Sessions d'étude - Société canadienne d'histoire de l'Église catholique, 41, 61-85. https://doi.org/10.7202/1007242ar

Tous droits réservés @ Ces Éditions Historia Ecclesiæ Catholicæ Canadensis Inc., 1975
Ce document est protégé par la loi sur le droit d'auteur. L'utilisation des services d'Érudit (y compris la reproduction) est assujettie à sa politique d'utilisation que vous pouvez consulter en ligne.

https://apropos.erudit.org/fr/usagers/politique-dutilisation/ 


\section{L'Église et l'argent : les quêtes commandées dans le diocèse de Sherbrooke, 1893-1926}

L'Église et l'argent: voilà un domaine, dans l'histoire de l'Église québécoise, qui a suscité jusqu'ici bien peu d'études et qui, pourtant, constitue un élément important du débat sur le rôle de l'Église dans la société canadienne-française. En 1971, le rapport Dumont a levé le coin du voile sur la situation financière de l'Église au Québec, en présentant les sources de financement et la répartition des dépenses des paroisses et des diocèses ${ }^{1}$.

Du côté des historiens de métier, on relève quelques travaux qui abordent la question de façon indirecte. C'est le problème des dîmes qui a le plus retenu l'attention. En 1958, l'abbé Proulx a présenté une thèse de licence sur le paiement de la dîme après la Conquête ${ }^{2}$; le produit des dîmes a été utilisé de manière très intéressante par Fernand Ouellet pour mesurer la production agricole ${ }^{3}$; enfin, le débat qu'a suscité la perception de la dîme au milieu du $\mathrm{XIX}^{\mathrm{e}}$ siècle a été brièvement analysé par Jean-Paul Bernard dans son volume sur Les Rouges 4 . Un autre problème à implication financière a beaucoup agité l'opinion publique : la question des Biens des Jésuites, qui a fait l'objet d'une excellente étude de R.C. Dalton ${ }^{5}$. Enfin, pour le $\mathrm{XX}^{\mathrm{e}}$ siècle, on ne trouve guère à signaler que le volume de W.F. Ryan, sur le clergé et la croissance

1 COMMISSION D'ÉTUde SUR LES LAÏCS ET L'ÉGLISE, L'Église du Québec : un héritage, un projet, Montréal, Fides, 1971. Appendice I : La question financière dans l'Église québécoise, pp. 295-315.

2 Georges-Etienne Proulx, «Les Canadiens ont-ils payé la dîme entre 1760 et 1775 ? », in R.H.A.F., 11 (1958), p.p. 533-562.

3 Fernand OUELLET, "L'agriculture bas-canadienne vue à travers les dîmes et la rente en nature », in Histoire sociale/Social History, no 8 (1971), pp. 5-44.

4 Jean-Paul Bernard, Les Rouges, Montréal, P.U.Q., 1971. Voir les pp. 77-79.

5 Roy C. Dalton, The Jesuits' Estates Question, 1760-1888. A Study of the Background to the Agitation of 1889, Toronto, Univ. of Toronto Press, 1968, 201 p. Voir aussi J.R. MILLR, The Impact of the Jesuits' Estates Act on Canadian Politics, 1888-1891, Ph.D. Thesis, Univ. of Toronto, 1972. 
économique du Québec de 1896 à $1914^{6}$, mais cette étude ne contient aucune donnée sur les biens ou la richesse de l’Église.

Il est donc étonnant qu'une question aussi controversée n'ait encore suscité aucune étude sérieuse, alors même que, grâce à la collaboration des archivistes diocésains ${ }^{7}$, les sources deviennent de plus en plus accessibles. La question peut être examinée sous deux angles. D'abord, on peut se placer du point de vue de l'institution et se demander quels sont les biens, meubles et immeubles, dont dispose l'Église, comment ils sont administrés, etc. On peut également aborder le problème à partir des contributions des fidèles, en examinant les sommes versées à l'Église sous forme de dîmes, de quêtes, de souscriptions, de répartitions.

C'est sur ce deuxième aspect que nous avons fait porter notre travail, en abordant la question des quêtes commandées dans le diocèse de Sherbrooke. Nous étudions ces quêtes à partir d'une source abondante et facile d'accès : le Rapport des œuvres diocésaines, publié chaque année dans le recueil des Mandements, lettres pastorales, circulaires et autres documents ${ }^{8}$. Nous avons là une série de données chiffrées, remarquable par sa continuité et par sa précision puisque le produit de chacune des quêtes est indiqué pour chaque paroisse du diocèse.

Comme limites chronologiques de notre étude, nous avons retenu les dates 1893 et 1926, début et terme de l'épiscopat de $\mathbf{M}^{\mathrm{gr}}$ Paul LaRocque, deuxième évêque de Sherbrooke. Comme la plupart des quêtes considérées sont commandées par l'évêque, il est utile d'envisager un long épiscopat, ce qui nous assure, éventuellement, une certaine unité d'action et de politique. Cette période coïncide avec un important développement de la population, de l'économie et des institutions des Cantons de l'Est. Il sera dès lors intéressant de suivre les variations du produit de ces quêtes. Enfin, nous exami-

6 William F. RyaN, The Clergy and Economic Growth in Quebec (1896-1914), Québec, P.U.L., 1966, 348p.

7 Nous tenons à remercier M. Gaston Provençal, archiviste diocésain de Sherbrooke, de sa grande disponibilité à notre égard.

8 Mandements, lettres pastorales, circulaires et autres documents publiés dans le diocèse de Sherbrooke, Sherbrooke, Imprimerie de «Le Progrès de l'Est 》, puis Imprimerie du 《Messager de Saint-Michel ». Nous avons utilisé les tomes IV à IX : t. IV (1893-1900), 403p.; t. V (1901-1909), 473p.; t. VI (1909-1915), 367p.; t. VII (1915-1919), numéroté 369-577; t. VIII (19201925), 520p.; t. IX (1926), 152p. Désigné désormais par Mand. Sherbrooke. 
nerons les facteurs qui pourraient expliquer ces variations, ce qui nous amènera non seulement à des considérations d'ordre économique, mais également à une analyse des mentalités, peut-être même de la spiritualité des pasteurs et de la population de ce diocèse au début du siècle.

\section{Les diverses quêtes commandées}

Il importe en premier lieu de préciser ce que nous entendons par l'expression "quêtes commandées 》. Nous en donnons une définition restrictive : il s'agit de quêtes ordonnées par l'évêque ou le pape, qui se font le même jour dans toutes les églises du diocèse 9 . Nous excluons de la sorte les souscriptions et les aumônes recueillies dans les troncs, en particulier les Aumônes de Carême, qui seront introduites en 1904 dans le diocèse de Sherbrooke et rapporteront des montants considérables, appliqués par l'évêque aux œuvres diocésaines ${ }^{10}$. Nous excluons également des œuvres comme la Saint-François de Sales ou la Propagation de la Foi, qui recueillent un montant fixe auprès de chaque associé ${ }^{11}$.

Quelles sont donc, au sens restreint où nous l'entendons, les diverses quêtes commandées qui ont été établies dans le diocèse de Sherbrooke ? A la mort de $\mathbf{M}^{\mathrm{gr}}$ Racine en 1893 , on en comptait six : l'Guvre du Séminaire, la plus ancienne (1875), et de loin la plus importante; puis, établies en 1882, les quêtes pontificales de la Terre Sainte (Vendredi-Saint) et du Denier de Saint-Pierre ${ }^{12}$, ainsi que les quêtes diocésaines pour la colonisation (24 juin) et pour les écoles pauvres du diocèse (Pentecôte); enfin, en 1891, la quête pontificale pour les Missions d'Afrique (Épiphanie), destinée à appuyer l'Guvre anti-esclavagiste du cardinal Lavigerie ${ }^{13}$.

9 Certaines de ces quêtes peuvent également être ordonnées par les évêques du Canada, par ceux du Québec, ou par ceux de la province ecclésiastique (Montréal).

10 L'étude du produit de ces "Aumônes quadragésimales》 serait très intéressante. L'évêque les recommande chaque année, et l'étude de ces Circulaires apporterait beaucoup, particulièrement sur la spiritualité du Carême (prière, jeûne, aumône).

11 L'CEuvre de la Propagation de la Foi sera relancée avec une nouvelle vigueur au début de 1922; cf. Mand. Sherbrooke, t. VIII, pp. 152-155.

12 "Mandement de Mgr A. Racine, établissant le Denier de Saint Pierre dans le Diocèse de Sherbrooke », in Mand. Sherbrooke, t. II, pp. 235-240.

13 Voir Circulaire du 15 janvier 1891, in Mand. Sherbrooke, t. III, pp. 227-231. 
Dès le début de l'épiscopat de $\mathrm{M}^{\mathrm{gr}} \mathrm{LaRocque}$ se produit un changement d'importance : la quête pour le Séminaire est abolie 14 et remplacée par deux quêtes annuelles pour l'Université Laval (succursale de Montréal) ${ }^{15}$. Nous avons donc là un total de sept quêtes qui auront lieu chaque année de 1895 à 1919. Il s'en ajoutera une nouvelle de 1910 à 1919, alors que les Pères du Premier Concile Plénier de Québec décideront de venir en aide aux Ruthènes (Ukrainiens) de l'Ouest canadien ${ }^{16}$. Il faut enfin noter, avant 1914, trois quêtes spéciales à l'occasion de sinistres : en 1900, à la suite de l'incendie de Hull; en 1909, pour secourir les victimes du tremblement de terre en Sicile et en Calabre, et en 1912, pour aider à la reconstruction du Séminaire et de la Cathédrale de Chicoutimi, disparus dans l'incendie de la ville ${ }^{17}$.

Durant la Grande Guerre, on retrouve deux quêtes spéciales directement reliées au conflit ${ }^{18}$, sans compter les encouragements fournis du haut de la chaire aux souscriptions du Fonds patriotique ${ }^{19}$. Signalons également une quête qui rapportera la plus forte somme jamais obtenue jusque-là dans le diocèse; il s'agit de la quête pour les Écoles de l'Ontario, que $\mathrm{M}^{\mathrm{gr}}$ LaRocque annonce ainsi : "Pour nos frères, les blessés de l'Ontario 20 ». Enfin, pour souligner l'intérêt que porte le diocèse à l'agriculture et à la colonisation, mentionnons la collecte spéciale pour le Monument Hébert, à l'occasion du tricentenaire de $1917^{21}$.

14 Mand. Sherbrooke, t. IV, p. 52.

15 Mandement de Mgr l'Archevêque de Montréal et de Nos Seigneurs les Évêques de la Province Ecclésiastique de Montréal recommandant l'Euvre de l'Université, octobre 1894, 6p.; Mand. Sherbrooke, t. IV, pp. 61-62.

À la même époque, la quête de l'CEuvre de la colonisation devient la «quête de l'œuvre des missionnaires agricoles et de la colonisation 》. Mand. Sherbrooke, t. IV, pp. 30-31.

16 Mand. Sherbrooke, t. VI, pp. 78-81.

17 Mand. Sherbrooke, t. IV, pp. 381-383; t. VI, pp. 4-6 et 13-15, pp. 211-214 et 216-217.

18 Il s'agit d'une collecte pour les Marins Britanniques, le 24 décembre 1916 (Mand. Sherbrooke, t. VII, pp. 442-444) et d'une quête pour les Huttes Catholiques de l'armée, le 22 septembre 1918 (Mand. Sherbrooke, t. VII, pp. 521-523).

19 Voir, par exemple, Mand. Sherbrooke, t. VII, pp. 395-397 et 401-402.

20 Circulaire du 19 février 1916, in Mand. Sherbrooke, t. VII, pp. $405-$ 409 et 422-423. Voir aussi Lettre de Mgr P. LaRocque au sénateur P. Landry, 6 avril 1916, in Archives de l'Archidiocèse de Sherbrooke, III-A-2.

21 Mand. Sherbrooke, t. VII, pp. 471-475. 
C'est après la guerre, et particulièrement durant les années 1920-1924, que les quêtes commandées connaîtront à Sherbrooke un développement extraordinaire. Le fait est d'autant plus remarquable que sévit au même moment une crise économique brève, mais aiguë. Mentionnons en premier lieu les quêtes nouvelles. Pour remplacer l'Guvre des Ruthènes, qui prenait fin en 1919, l'évêque ordonne d'abord une quête pour l'Euvre bénédictine de SaintBenoît-du-Lac ${ }^{22}$, puis il institue l'Euvre des Vocations Sacerdotales, pour laquelle il y aura une quête annuelle ${ }^{23}$. De même, à la suite de la grande souscription publique de 1920 en faveur de l'Université de Montréal devenue complètement autonome ${ }^{24}$, les quêtes pour l'Université sont remplacées par deux nouvelles quêtes : l'une pour une communauté qui venait d'être fondée dans le diocèse, les Sæeurs missionnaires de Notre-Dame des Anges, et l'autre, pour venir en aide aux curés des paroisses pauvres du diocèse ${ }^{25}$. Deux autres quêtes annuelles apparaîtront en 1924 : celle du 24 juin, au profit d'une maison d'été pour les séminaristes, qui remplace la quête des missionnaires agricoles ${ }^{26}$, et celle du $1^{\text {er }}$ dimanche de décembre, pour les « œuvres catholiques», entendez les syndicats catholiques du diocèse ${ }^{27}$. À la mort de $\mathrm{M}^{\mathrm{gr}}$ LaRocque en 1926, le diocèse de Sherbrooke compte donc neuf quêtes commandées régulières, dont cinq instituées sous son épiscopat. Le Tableau I fournit une vue d'ensemble de ces quêtes, avec leur durée respective.

22 Circulaire du 24 novembre 1919, in Mand. Sherbrooke, t. VII, pp. 567-573.

23 Mand. Sherbrooke, t. VIII, pp. 43-48.

24 Mand. Sherbrooke, t. VIII, pp. 13-16.

25 Mand. Sherbrooke, t. VIII, pp. 295-298. L'évêque s'explique ainsi de cette dernière quête, en faveur des paroisses et missions pauvres du diocèse : «Jusqu'en ces derniers temps les offrandes substantielles de la Saint-François de Sales permettaient à l'Évêque d'aider matériellement ces prêtres; mais depuis l'organisation de l'Euvre de la Propagation de la Foi dont les recettes sont envoyées à Rome, ces offrandes sont complètement disparues 》. Ibid., p. 297.

Mand. Sherbrooke, t. VIII, pp. 436-440. 
Durée des quêtes commandées, diocèse de Sherbrooke, 1894-1926

A- Quêtes annuelles

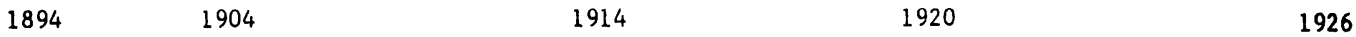

(1881) Missionnaires agricoles

(1882) Terre Sainte

(1882) Denier de S. Plerre

Ecoles pauvres

(1891) Missions d'Afrique

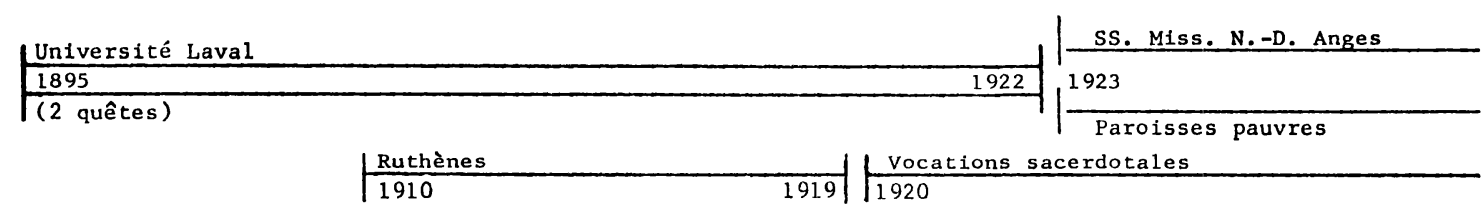

B- Quêtes spéciales

$\begin{array}{ccccccccc}\text { B- Quêtes } & \text { spéciales } \\ 1900 & 1909 & 1912 & 1916 & 1917 & 1918 & 1919 & 1921 & 1922 \\ X & X & X & X & X & X & X & X & X \\ & & & X & & & & & X \\ \end{array}$

Oeuvres catholiques

1924

N.B. Par souc1 de clarté, la ligne du temps n'est pas à l'échelle.

Les dates entre parenthèses indiquent l'année où ont commencé les quêtes qui existalent avant 1894. 
Deux constantes se dégagent de cette présentation. Quand une quête est abandonnée, pour une raison ou pour une autre, elle est aussitôt remplacée par une nouvelle quête, répondant à un nouveau besoin. Deuxièmement, on peut dire que les nouvelles quêtes reflètent les grandes préoccupations de l'Église, soit au niveau universel, où l'élan donné par Benoît XV et Pie XI à la formation du clergé et au développement des missions trouve ici son écho, soit au niveau du Québec, comme on le voit dans le cas des syndicats catholiques. Disons enfin que les quêtes spéciales des années d'après-guerre connaîtront un grand succès. Il s'agit de trois quêtes commandées par le Souverain Pontife pour les victimes de guerre en 1921 et $1922^{28}$, de la collecte pour la reconstruction de la Basilique de Sainte-Anne de Beaupré à la suite de l'incendie de $1922^{29}$, et d'une ultime quête pour les Ruthènes ${ }^{30}$.

Il faut ajouter que les quêtes commandées ne sont pas le seul moyen de pourvoir aux œuvres du diocèse. Ainsi, en 1898, pour financer la reconstruction de son Séminaire incendié, l'évêque convoque une réunion du clergé, où il est décidé d'utiliser les revenus de l'EEuvre de Saint-François de Sales et de prélever $10 \%$ des rentes des bancs durant cinq ans ${ }^{31}$. Les cinq ans écoulés, l'évêque retient $5 \%$ sur le revenu des bancs pour établir l'Euvre de la Cathédrale ${ }^{32}$. En 1920, c'est à de grandes souscriptions qu'on recourt, que ce soit pour l'Université de Montréal ou pour l'Hospice du Sacré-Couur ${ }^{33}$. Les quêtes commandées que nous étudions ne sont donc pas les seuls moyens extraordinaires de financement des œuvres utilisés par l'Eglise; elles en constituent cependant une part très importante.

28 Circulaire du 2 janvier 1921, prescrivant une quête pour les «Orphelins de la guerre», in Mand. Sherbrooke, t. VIII, pp. 70-71 et 80; Circulaire du 2 janvier 1922, pour les «Enfants affamés d'Europe Centrale et Orientale », in Mand. Sherbrooke, t. VIII, pp. 160-164 et 189; Circulaire du 15 octobre 1922, pour les populations de Russie, in Mand. Sherbrooke, t. VIII, pp. 228-232.

29 Circulaire du 24 juillet 1922, in Mand. Sherbrooke, t. VIII, pp. 223225.

30 Circulaire du 5 août 1925, in Mand. Sherbrooke, t. VIII, pp. 491-495.

31 Lors de cette réunion, le clergé avait aussi suggéré d'utiliser le produit des deux quêtes pontificales des Missions d'Afrique et de Terre Sainte - ce qui donne une idée de l'intérêt qu'il portait à ces œuvres... Le Préfet de la Propagande opposa un refus à cette demande. Mand. Sherbrooke, t. IV, pp. 265-272.

32 Mand. Sherbrooke, t. V, pp. 223-226 et 286-288. Voir aussi t. VIII, p. 298.

33 Mand. Sherbrooke, t. VIII, pp. 13-16 et 24-28. 
TABLEAU II

Produit des quêtes commandées, diocèse de Sherbrooke, 1894-1926

\begin{tabular}{|c|c|c|c|c|c|c|c|c|c|}
\hline ANNEE & $\begin{array}{l}\text { MISSIONS } \\
\text { D'AFRIQUE }\end{array}$ & $\begin{array}{l}\text { TERRE } \\
\text { SAINTE }\end{array}$ & $\begin{array}{l}\text { DENIER } \\
\text { S.PIERRE }\end{array}$ & $\begin{array}{l}\text { UNIV. LAVAL } \\
2 \text { quêtes. }\end{array}$ & $\begin{array}{l}\text { ECOLES } \\
\text { PAUVRES }\end{array}$ & $\begin{array}{l}\text { MISS. } \\
\text { AGRICOLES }\end{array}$ & RUTHENES & TOTAL & \multirow{17}{*}{ 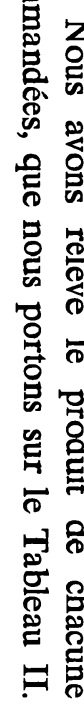 } \\
\hline 1894 & $\$ 146.50$ & $\$ 182.11$ & $\$ 175.25$ & & $\$ 174.09$ & $\$ 147.80$ & & $\$ 825.75$ & \\
\hline 1895 & 151.09 & 188.52 & 161.80 & 341.16 & 148.50 & 129.42 & & 1020.49 & \\
\hline 1896 & 147.18 & 156.07 & 176.38 & 317.63 & 174.62 & 144.62 & & 1116.50 & \\
\hline *1897 & 154.11 & 176.38 & 203.35 & $\left\{\begin{array}{l}152.83 \\
188.39\end{array}\right.$ & 187.34 & 137.51 & & 1199.91 & \\
\hline *1898 & 174.84 & 196.62 & 222.33 & 326.65 & 183.77 & 138.05 & & 1242.26 & \\
\hline 1899 & 139.61 & 166.85 & 200.58 & 320.30 & 153.71 & 148.74 & & 1129.79 & \\
\hline *1900 & 159.93 & 167.53 & 199.18 & 334.50 & 177.78 & 157.01 & & 1195.93 & \\
\hline 1901 & 162.29 & 186.97 & 207.08 & 336.92 & 175.07 & 141.82 & & 1210.15 & \\
\hline *1902 & 207.83 & 208.00 & 234.36 & $\left\{\begin{array}{l}190.17 \\
206.04\end{array}\right.$ & 227.75 & 180.49 & & 1454.64 & \\
\hline 1903 & 198.43 & 245.87 & 279.08 & 403.87 & 218.75 & 209.54 & & 1555.54 & \\
\hline 1904 & 200.20 & 220.66 & 254.25 & 378.99 & 227.24 & 173.28 & & 1454.62 & \\
\hline 1905 & 184.45 & 252.77 & 285.75 & 416.40 & 200.01 & 179.83 & & 1519.21 & \\
\hline 1906 & 255.83 & 298.73 & 333.55 & 501.91 & 278.26 & 255.53 & & 1923.81 & \\
\hline 1907 & 253.67 & 299.19 & 409.32 & 477.47 & 284.90 & 256.40 & & 1980.95 & \\
\hline 1908 & 266.84 & +355.55 & 446.50 & +512.25 & 295.36 & 252.08 & & 2128.58 & \\
\hline 1909 & 263.24 & 318.67 & 393.31 & +465.94 & 275.49 & 250.66 & & 1967.31 & \\
\hline 1910 & 231.08 & 353.36 & 407.66 & 507.10 & 292.12 & 242.97 & 530.61 & 2564.90 & คి \\
\hline 1911 & 264.41 & 331.36 & 453.44 & 551.87 & 296.06 & 272.91 & 495.75 & 2575.80 & \\
\hline $\begin{array}{l}1912 \\
1913 \\
1914\end{array}$ & $\begin{array}{l}256.83 \\
322.97 \\
340.26\end{array}$ & $\begin{array}{l}385.43 \\
377.99 \\
421.71\end{array}$ & $\begin{array}{l}469.70 \\
461.62 \\
500.40\end{array}$ & $\begin{array}{l}529.91 \\
576.27 \\
600.72\end{array}$ & $\begin{array}{l}314.36 \\
316.67 \\
347.73\end{array}$ & $\begin{array}{l}300.72 \\
301.20 \\
322.04\end{array}$ & $\begin{array}{l}341.38 \\
382.93 \\
356.71\end{array}$ & $\begin{array}{l}2598.33 \\
2739.65 \\
2889.57\end{array}$ & \\
\hline 1915 & 340.53 & 428.38 & 480.25 & 560.09 & 342.37 & 295.92 & 291.96 & 2739.50 & \\
\hline 1916 & 329.50 & 438.18 & 559.25 & 705.31 & 370.36 & 327.57 & 339.16 & 3069.33 & Q \\
\hline 1917 & 372.63 & 435.65 & 556.58 & 743.85 & 427.08 & 373.00 & 390.32 & 3299.11 & 8 \\
\hline 1918 & 442.89 & 538.10 & 634.16 & 791.96 & 422.80 & 387.90 & 427.12 & 3644.93 & \\
\hline 1919 & 446.08 & 549.74 & 669.34 & 954.31 & 449.96 & 463.26 & 435.66 & 3968.35 & \\
\hline
\end{tabular}


UNIV. ECOLES MISS.

VOC. SS.MISS. PAROISSES OEUVRES

TOTAL

\begin{tabular}{|c|c|c|c|c|c|c|c|c|c|c|c|}
\hline 1920 & 539.66 & 713.69 & 951.30 & 1174.90 & 675.30 & 538.20 & 2030.85 & & & & 6623.90 \\
\hline 1921 & 793.28 & 957.17 & 1185.19 & 1202.64 & 722.62 & 629.96 & 1331.39 & & & & 6822.25 \\
\hline${ }^{*} 1922$ & 815.69 & 872.28 & 1192.27 & 1113.08 & 616.61 & 559.98 & 1417.35 & & & & 6587.26 \\
\hline *1923 & 778.37 & 972.62 & 1067.25 & & 836.90 & 718.75 & 1476.74 & 1145.23 & 1118.31 & & 8114.17 \\
\hline 1924 & \multicolumn{10}{|c|}{ MAISON D'ETE } & 10018.30 \\
\hline *1925 & 968.94 & 1056.68 & 1354.12 & & 952.55 & 939.18 & 1146.27 & 1038.87 & 897.39 & 867.14 & 9221.14 \\
\hline *1926 & 1069.66 & 1108.09 & 1243.16 & & 903.62 & 966.28 & 1185.92 & 1087.02 & 1016.46 & 824.48 & 9404.69 \\
\hline
\end{tabular}

\section{Produit des quêtes spéciales:}

\section{mai 1900}

fév. 1909

juil. 1912

fév. 1916

déc. 1916

juin 1917

sept. 1918

déc. 1919

j anv. 1921

janv. 1922

sept. 1922

déc. 1922

sept. 1925

Incendie de Hull

Secours aux Siciliens et Calabrais

Cathédrale et Séminaire de Chicoutimi

Ecoles d'Ontario

Marins Britanniques

Monument Hébert

Huttes Catholiques de l'armée

Oeuvre de S. Benost du Lac

Orphelins de la guerre

Enfants affamés d'Europe Centrale

Basilique Ste-Anne de Beaupré

Enfants de Russie

Ruthènes (estimé: 400.00)

1027.16

964.82

2132.08

683.31

536.33

(estimé: 800.00 )

(estimé: 1600.00)

3060.55

3812.28

11076.69

2342.23

2425.00 (montant envoyé)

* Indique que pour l'année en question, la compilation du total de chaque quête est de nous.

+ Indique un total corrigé par nous.

Source: Rapport annuel des Oeuvres diocésaines, in Mand. Sherbrooke, 1894-1926. 
Une mise en garde s'impose au départ : il est impossible, dans l'état actuel de la documentation, d'être assuré de l'exactitude de chaque chiffre. Nous n'avons pu retrouver aux Archives diocésaines les données de base qui ont servi à publier le Rapport des Euvres. Les nombreuses vérifications que nous avons effectuées montrent que le produit total d'une quête n'est pas toujours égal au total des sommes recueillies dans chaque paroisse. Il reste que les erreurs sont habituellement inférieures à $5 \%$ et que les curés auraient fait apporter

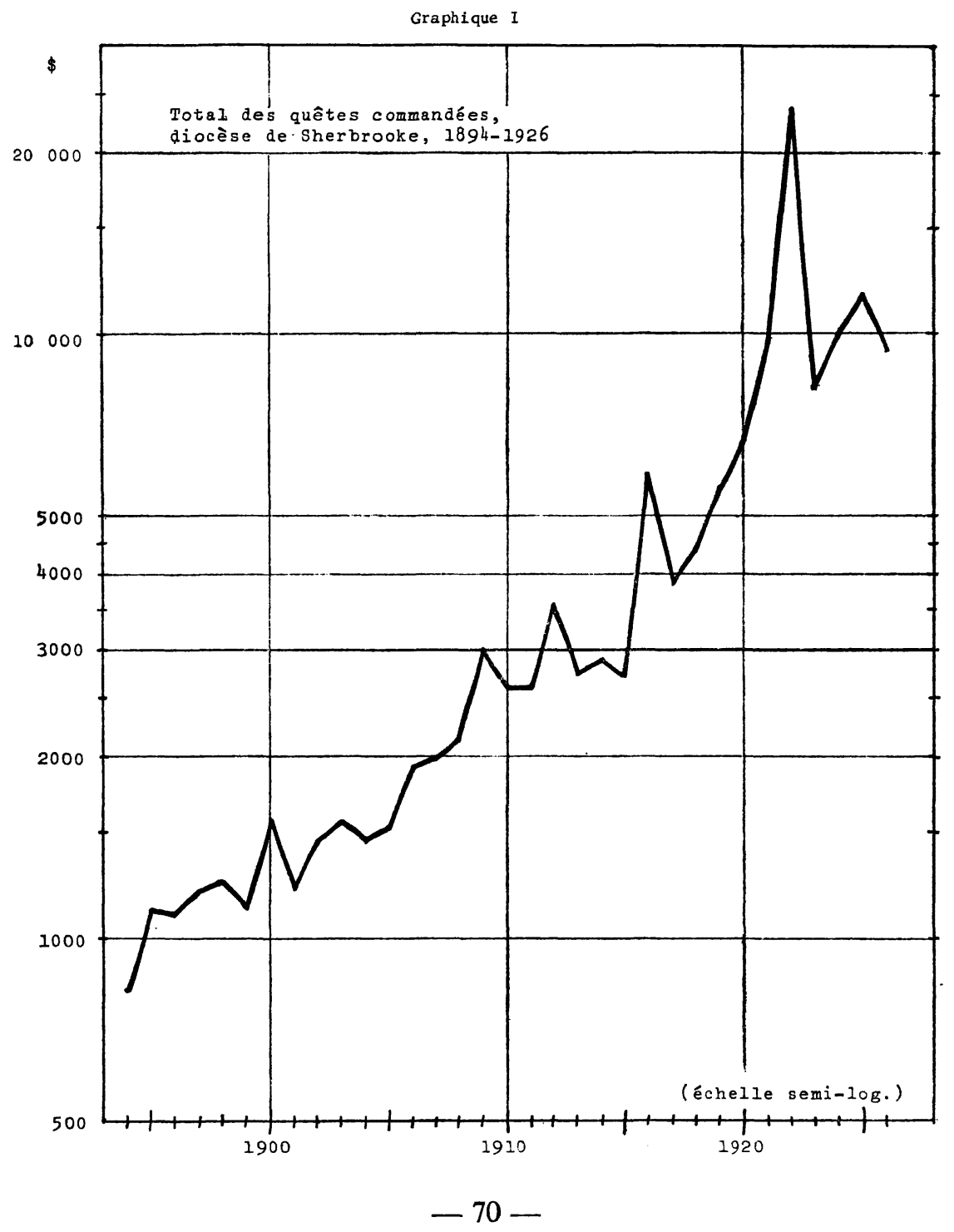


Graphique II

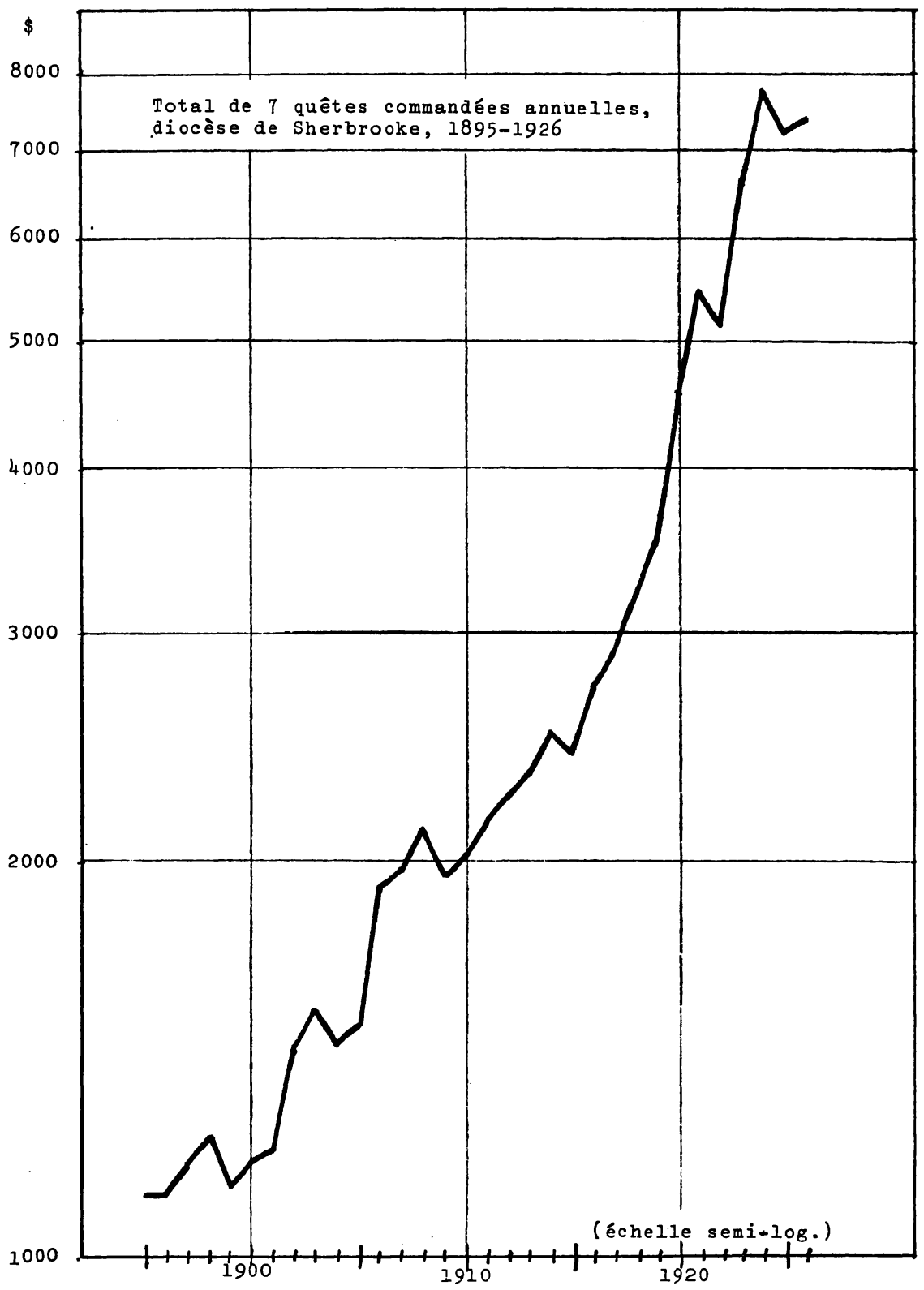


des corrections si une erreur trop importante s'était glissée dans le Rapport ${ }^{34}$.

Ceci dit, examinons le total annuel de l'ensemble des quêtes commandées (Graphique I). On note d'abord une croissance constante, avec certains sommets qui s'expliquent facilement soit par de nouvelles quêtes qui font leur apparition (1895), soit par des quêtes spéciales qui sont commandées $(1900,1909,1912,1916$, $1922,1925)^{35}$.

Pour avoir une meilleure idée de l'évolution du produit des quêtes durant ces 33 ans, il est donc nécessaire de défalquer les quêtes spéciales et de retenir un nombre égal de quêtes pour chaque année. C'est ce que nous faisons sur le Graphique II, qui indique le total de sept quêtes effectuées chaque année de 1895 à $1926^{36}$.

On peut alors distinguer deux périodes. Jusqu'en 1915, la croissance est lente, mais régulière : on passe, en gros, de $\$ 1000$. en 1895 à $\$ 2500$. en 1914. À partir de 1915 , la croissance s'accélère : de $\$ 2500$. en 1915 , le produit des quêtes retenues s'élève à plus de $\$ 7000$. en 1926. Il est donc remarquable que c'est au moment même où de nombreuses quêtes spéciales viennent s'ajouter aux quêtes commandées annuelles (1916-1925) que ces dernières connaissent leur taux de croissance le plus élevé.

Comment expliquer cette croissance? Plusieurs hypothèses peuvent être envisagées. On peut invoquer le développement général de la région, une meilleure organisation des paroisses, la générosité accrue des fidèles. Mais avant d'examiner ces facteurs qualitatifs, il importe de vérifier certaines données strictement quantitatives.

34 Dans le cas de quatre quêtes spéciales, le Rapport ne fournit pas de résultats. Dans l'un de ces cas, nous avons pu retrouver l'indication du montant dans les Archives (Archives de l'Archevêché de Sherbrooke, II-G, SaintBoniface, Rapport de la Quête spéciale faite en 1924-1926, pour les œuvres catholiques des Ruthènes au Canada).

Dans les trois autres cas (20 mai 1900 : Hull; 22 septembre 1918 : Huttes Catholiques de l'armée; 7 décembre 1919 : S. Benoît du Lac), nous avons dû nous-même faire un estimé à partir du résultat de quêtes similaires à la même époque.

35 À noter que pour chaque année sans exception de 1916 à 1925, il y a soit une quête spéciale, soit une nouvelle quête; en 1922, il y a trois quêtes spéciales.

36 Nous laissons donc de côté les quêtes annuelles pour les Ruthènes, pour les Vocations sacerdotales et pour les Euvres catholiques. 


\section{Les facteurs quantitatifs de variation}

Tout d'abord, l'accroissement démographique. Si la population a augmenté, on ne s'étonnera pas de voir le produit des quêtes augmenter. Mais il n'est pas facile de reconstituer le chiffre de la population catholique du diocèse. La source la plus complète à cet égard est Le Canada Ecclésiastique, publié depuis 1887, qui fournit chaque année le chiffre de la population catholique du diocèse. Mais ce chiffre n'est pas revu tous les ans. Ainsi, de 1909 à 1915, c'est le même chiffre de 85000 catholiques qui est toujours mentionné. Nous n'avons donc retenu le chiffre du Canada Ecclésiastique que pour les années où il variait, soit pour un total de douze années (1895, 1896, 1899, 1905, 1908, 1909, 1916, 1918, 1920, 1922, $1924,1925)$.

Nous avons ensuite consulté les recensements décennaux de 1901, 1911 et 1921. Mais là, les chiffres sont fournis par comté. Comme les frontières du diocèse ne coïncident pas toujours avec celle des comtés, il nous a fallu reconstituer le nombre de catholiques de certaines parties du diocèse par village et par canton. Nous obtenons ainsi trois chiffres, dont le premier, celui de 1901, est un peu plus élevé que celui du Canada Ecclésiastique, alors que les deux autres cadrent parfaitement avec les données de cet annuaire ${ }^{37}$.

Enfin, sans que nous ayons pu les critiquer suffisamment, nous avons consulté les chiffres fournis dans les différentes « Relations 》 que l'évêché envoyait régulièrement au Saint-Siège ${ }^{38}$. Ils confirment à peu près les chiffres précédemment établis.

Au total, on constate que la population catholique du diocèse croît de façon régulière, passant en trente ans de 60000 à plus de 100000 habitants ${ }^{39}$. En divisant le produit des quêtes annuelles

37 Recensements du Canada, 1901, vol. I, pp. 226-268; 1911, vol. II, pp. 88-135; 1921, vol. I, pp. 626-671. Les chiffres obtenus sont les suivants : 1901 : 71555 catholiques; 1911 : 88359 catholiques; 1921 : 107898 catholiques.

38 Archives de l'Archidiocèse de Sherbrooke, Section IV-C, Visites ad limina. Voici les chiffres fournis : 1885 : 46500 catholiques; 1891 : 53 276; 1896: environ 60000 ; 1904 : environ 76000 catholiques; 1914 : 95 000; 1919 : $100000 ; 1924: 105000$.

39 Notons que durant la même période, le nombre de protestants décroît, particulièrement de 1911 à 1921. 


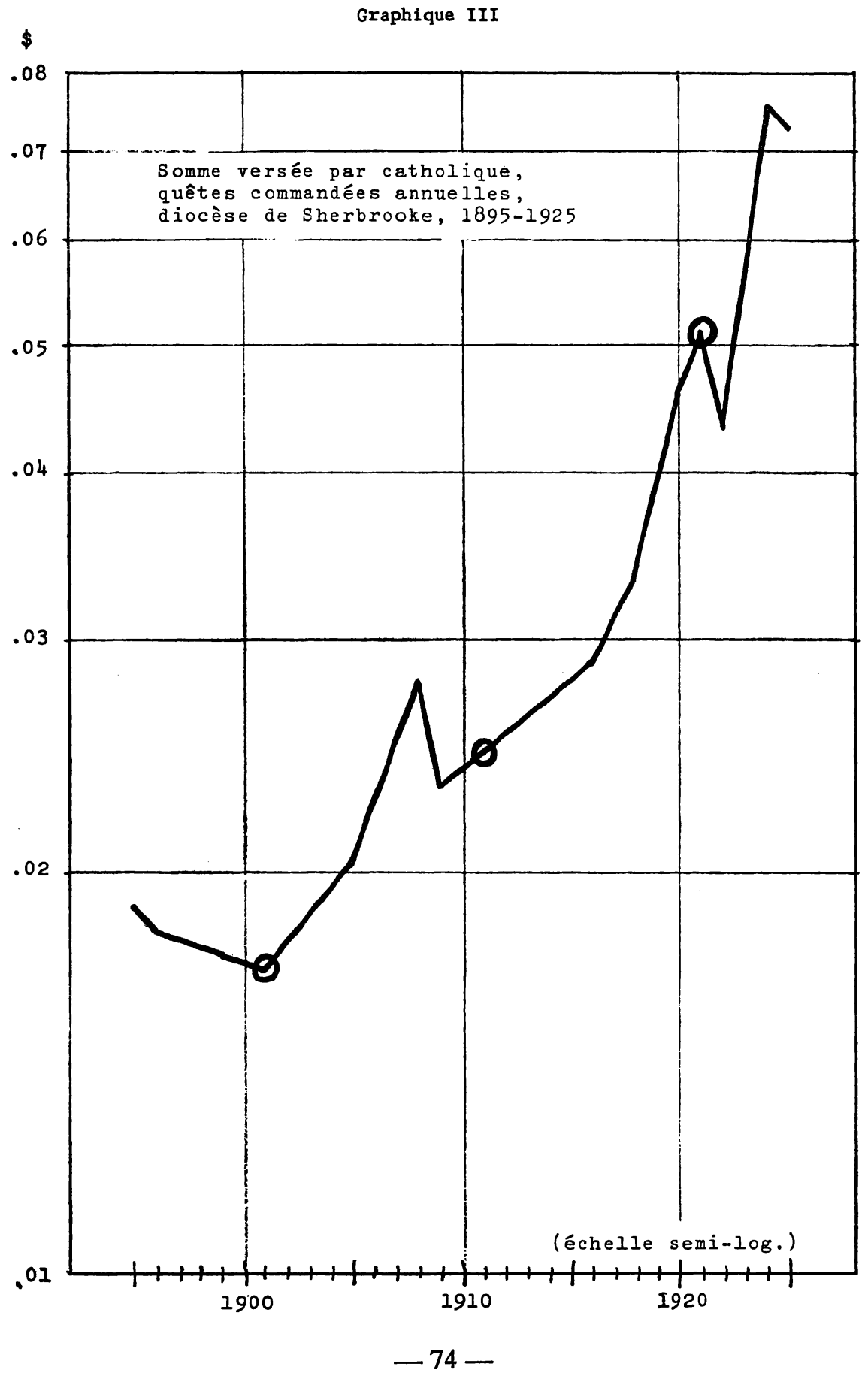


régulières par le nombre de catholiques ${ }^{40}$, on obtient une nouvelle courbe (Graphique III), qui confirme le phénomène déjà observé : croissance régulière durant toute la période, avec accélération à partir de 1915. En gros, les sept quêtes commandées régulières ont rapporté $2 \phi$ par catholique de 1895 à $1905,3 \phi$ vers 1915 et entre 5 et $7 \not$ de 1920 à 1925.

Le deuxième facteur qu'il nous faut examiner est d'ordre économique ${ }^{41}$. On peut utiliser plusieurs critères pour tenter de mesurer l'influence de la conjoncture économique sur le produit des quêtes. Pour la période envisagée, les sources concernant directement la région qui nous intéresse sont rares. C'est pourquoi nous utilisons des données statistiques relatives à l'ensemble du Canada, en supposant que la région des Cantons de l'Est suit, pour l'essentiel, une évolution comparable à celle du pays.

On peut d'abord se demander dans quelle mesure le produit des quêtes est affecté par l'inflation, particulièrement forte de 1914 à 1920. Les hausses considérables que nous avons constatées pour cette période ne seraient-elles dues qu'à la poussée inflationniste? Nous aurions voulu prendre comme indice la variation des prix des produits à la consommation. Mais cette donnée n'est disponible qu'à partir de 1913. Nous avons donc choisi l'indice des prix de gros, avec comme base $1926=100^{42}$. Les traits les plus marqués de cette courbe sont une hausse considérable des prix, de 65 en 1914 à 156 en 1920, suivie d'une chute à 97 lors de la crise de 1922.

Grâce à cet indice, on peut tracer la courbe de la variation du produit des sept quêtes annuelles considérées en dollars constants (Graphique IV). On constate alors que jusqu'en 1915, les courbes II et IV subissent des variations comparables, si l'on fait exception de deux baisses en dollars constants en 1907 et en 1912. Par contre, de 1915 à 1918, bien que les sommes recueillies augmentent en

40 Nous faisons cette opération à partir des seules douze années retenues dans Le Canada Ecclésiastique, auxquelles nous ajoutons les trois années du recensement fédéral, entourées d'un cercle.

41 Nous remercions vivement notre collègue Marc Vallières, à qui nous sommes particulièrement redevable pour cette section. Nous portons évidemment seul la responsabilité de nos avancés.

42 Canada. Dominion Bureau of Statistics, Prices and Price Indexes, 1913-1933, p. 15. L'indice des prix des produits à la consommation (ibid., p. 31) suit une courbe similaire. 


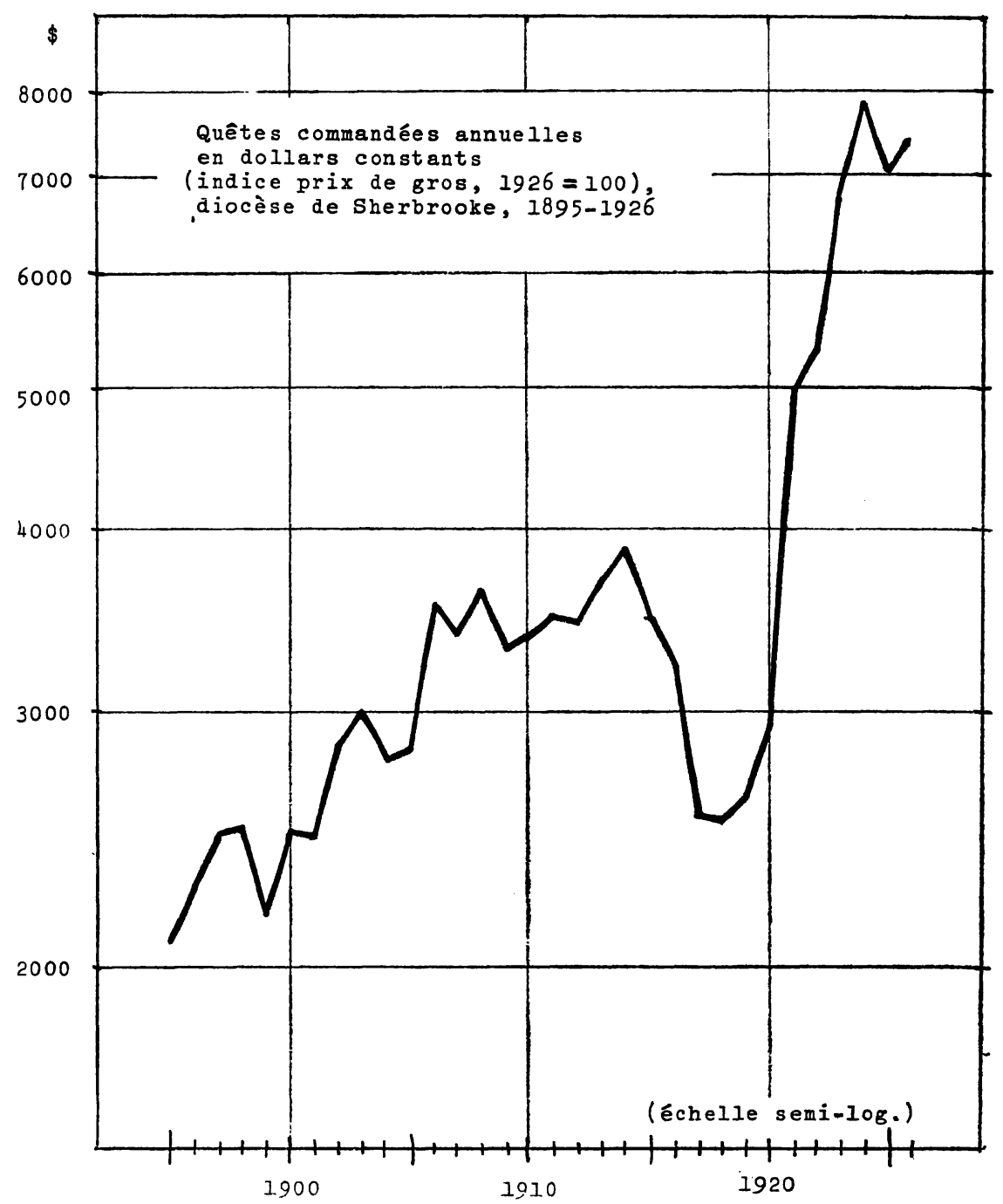

chiffres absolus (de $\$ 2450$. à $\$ 3200$.), elles diminuent considérablement en dollars constants, passant de $\$ 3475$. à $\$ 2525$., de telle sorte que le pouvoir d'achat résultant de ces quêtes s'en trouve sensiblement réduit. En revanche, en 1919 et en 1920, le produit des quêtes en dollars constants recommence à augmenter, alors 
même que l'inflation connaît son sommet. Et au moment de la crise aiguë de 1921-1922, le produit des quêtes continuera de croître en chiffres absolus, la légère chute de 1922 étant attribuable pour une large part à la tenue - exceptionnelle - de trois quêtes spéciales cette année-là. Il est donc indéniable qu'il y a, dans l'après-guerre, un essor considérable des contributions des fidèles aux quêtes commandées, qu'elles soient régulières ou spéciales.

On pourrait également, d'un point de vue économique, comparer le produit annuel des quêtes avec les revenus des habitants de la région. Mais, outre que les données sur les revenus sont difficiles à évaluer pour une région à cette époque - et encore plus difficiles lorsqu'on ne veut considérer que les seuls catholiques, les résultats qu'on obtiendrait seraient, à peu de choses près, les mêmes que ceux des autres indicateurs économiques ${ }^{43}$.

En somme, l'examen de sept quêtes commandées annuelles et l'analyse des courbes II-III-IV, qui introduisent des éléments de comparaison d'ordre démographique et économique, permettent de conclure à une croissance générale du produit des quêtes, mais avec certaines variations. Avant 1901, le produit diminue si l'on tient compte de l'accroissement de la population catholique. De 1901 à 1914 , les sommes recueillies augmentent régulièrement, malgré quelques baisses en 1904 et 1909. La période 1915-1918 est la plus délicate à interpréter : malgré une forte croissance en valeur absolue, le produit des quêtes subit un fléchissement important en dollars constants. La reprise de l'après-guerre est très nette sur tous les tableaux : même la crise de 1921-1922 ne permet pas d'entamer la forte croissance des sommes recueillies.

Nous avons tenté d'analyser avec rigueur les chiffres dont nous disposions, en gardant bien en mémoire la fragilité de nos données, tant pour le produit des quêtes que pour les indices qui nous ont servi de points de comparaison. Voyons maintenant si les facteurs d'ordre qualitatif pourront aider à expliquer cette croissance générale du produit des quêtes.

43 Nous avons effectué une vérification avec comme indice la moyenne des salaires pour diverses classes de travailleurs au Canada, de 1901 à 1926 (Wages and Hours of Labour in Canada, 1920-1929, p. 5). La courbe obtenue suit, en effet, avec des amplitudes quelque peu différentes, celle de l'indice des prix de gros. 


\section{Les facteurs qualitatifs de variation}

Le premier facteur qui vient à l'esprit et le plus naturel, en quelque sorte, est l'objet même de la quête. On donne davantage pour une œuvre qui apparaît comme plus importante. Cependant,

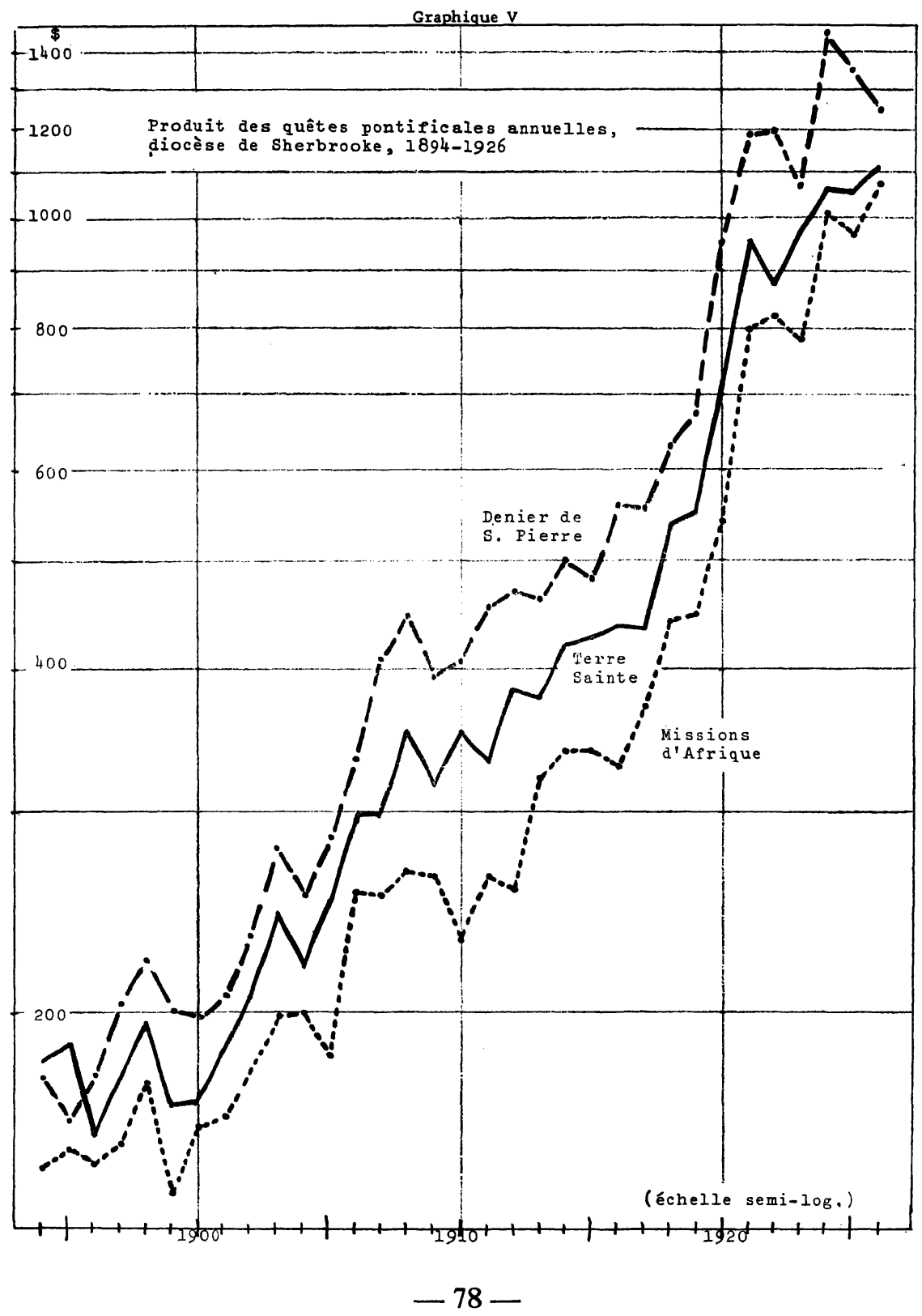


pour vérifier ce facteur, il faut le combiner avec la date de la quête : il n'est pas impossible que la même quête, faite à deux dates différentes, ne rapporte pas la même somme ${ }^{44}$. Nous trouvons une confirmation de ces deux premiers facteurs lorsque nous comparons le produit des trois quêtes pontificales annuelles: les Missions d'Afrique, la Terre-Sainte et le Denier de Saint-Pierre (Graphique V). Sauf pour les deux premières années (1894 et 1895), on vérifie dans tous les cas que la quête pour le Denier de Saint-Pierre rapporte plus que celle de la Terre-Sainte, et que cette dernière, à son tour, fournit de meilleurs résultats que celle des Missions d'Afrique. Il est donc clair d'après ce graphique que l'objet et la date de la quête influent sur son produit.

Une deuxième série de facteurs concernent la présentation de la quête par celui qui l'ordonne, à savoir l'évêque dans la plupart des cas. Les circulaires épiscopales abondent en recommandations de toutes sortes au sujet des quêtes commandées. Dès son arrivée dans le diocèse, $\mathbf{M}^{\mathrm{gr}} \mathrm{LaRocque}$ multiplie les reproches et les rappels à cet égard :

Je dois attirer votre attention sur le fait regrettable que quelques curés n'attachent pas aux quêtes ordonnées par Notre Saint Père le Pape ou par l'Évêque diocésain, l'importance qu'ils devraient. Vous ne trouverez pas inopportun que je vous dise franchement qu'il ne vous est pas loisible de faire ou d'omettre ces quêtes. Ayez soin de les annoncer d'avance afin qu'elles soient aussi abondantes que possible $[\ldots]^{43}$.

De manière générale, on peut dire que l'évêque veille à ce que les quêtes se fassent avec régularité et c'est sans doute un des facteurs qui expliquent la croissance du produit des quêtes.

44 Nous en avons un indice dans le cas des deux quêtes annuelles pour l'Université Laval. Dans deux cas (1897 et 1902), on nous fournit les résultats séparés pour les deux quêtes. Or, la quête de la Saint-Michel (fin septembre) rapporte plus que celle du $3^{\mathrm{e}}$ dimanche après Pâques (début mai), dans des proportions de 23 et $8 \%$.

45 Circulaire du 6 avril 1894, in Mand. Sherbrooke, t. IV, p. 27. Et encore le 4 novembre de la même année: "Vous ne devrez en omettre aucune sous quelque prétexte que ce soit; et si, dans un cas particulier, il y avait eu oubli, il faudrait invariablement reprendre cette quête le dimanche suivant ». Ibid., p. 62. 
Il est un cas où on peut particulièrement bien distinguer l'influence des exhortations épiscopales sur la sensibilité et la générosité des diocésains : c'est celui des quêtes faites pour une occasion spéciale, désastre, famine ou grande cause catholique. De 1894 à 1926, il y a treize quêtes de ce genre, et nous connaissons le produit de dix d'entre elles. Or ce produit est beaucoup plus élevé que celui des autres quêtes ordonnées, comme le montre le Tableau III.

Tableau III

Quêtes annuelles et quêtes spéciales

\begin{tabular}{l|c|rl}
\hline & $\begin{array}{c}\text { Produit moyen des } \\
\text { quêtes annuelles }\end{array}$ & \multicolumn{1}{|c}{$\begin{array}{c}\text { Produit des } \\
\text { quêtes spéciales }\end{array}$} \\
\hline 1909 & 281.04 & 1027.16 (Sicile) \\
1912 & 324.79 & 964.12 (Chicoutimi) \\
1916 & 383.66 & 2132.08 (Ontario) \\
& 683.31 (Marins britanniques) \\
1917 & 412.39 & 536.33 (Louis Hébert) \\
1921 & 852.78 & 3060.55 (Victimes de guerre) \\
1922 & 823.41 & 3812.28 (Europe Centrale) \\
& 11076.69 (Ste-Anne de Beaupré) \\
& 2342.23 (Russie) \\
1925 & 1024.57 & 2425. & (Ruthènes) \\
\hline
\end{tabular}

Chacune des quêtes spéciales produit beaucoup plus que la moyenne des quêtes commandées régulières de l'année correspondante, mais il y a de grandes variations d'une quête spéciale à l'autre. Ceci dépend de l'objet de la quête et des exhortations de l'évêque. Les deux quêtes pour les Marins britanniques et le Monument LouisHébert rapportent relativement beaucoup moins que les quêtes demandées par le Pape pour les Orphelins de la Grande Guerre, dont le produit est le triple de la moyenne des autres quêtes commandées. Notons enfin que deux quêtes ont connu un succès exceptionnel: celle des Écoles de l'Ontario, qui rapporte cinq fois plus que la moyenne, et celle de Sainte-Anne de Beaupré, dont le produit est treize fois plus élevé que la moyenne.

Ces résultats ne sont pas le fruit du hasard. Pour chacune de ces occasions, l'évêque a préparé une circulaire, qui doit être lue dans toutes les églises et commentée au besoin : 
Vous lirez la présente circulaire au prône dimanche prochain, 13 mai, en la commentant à votre gré afin de rendre aussi abondante que possible la quête qui devra se faire, dans toutes les églises du diocèse, le dimanche suivant, 20 mai, et dont le produit sera immédiatement transmis à l'évêché 46 .

Dans le cas des sinistres (incendie, famine), on décrit l'ampleur des dégâts et on mise sur la sensibilité des fidèles, particulièrement en ce qui concerne le triste sort des orphelins. On reproduit au besoin l'appel déchirant d'un témoin, comme dans le cas de la quête pour les Enfants affamés d'Europe Centrale et Orientale :

Ah! si les personnes charitables de l'Occident pouvaient voir, comme j'ai vu, de mes propres yeux, des petits enfants, moitiénus ou nus entièrement, nourris de détritus, de pelures, de feuilles d'arbres, d'herbes, même de vers de terre ... (etc.) ${ }^{47}$.

D'ailleurs, les résultats sont directement proportionnels à la chaleur de l'appel de l'évêque. Ceci est particulièrement visible dans le cas des Écoles de l'Ontario et pour la quête de Sainte-Anne de Beaupré. Dans le premier cas, après avoir cité une lettre éloquente du sénateur Landry, $\mathrm{M}^{\mathrm{gr}}$ LaRocque lance un appel pressant que les curés devront transmettre à leurs paroissiens : "Qu'ils comprennent bien que jamais peut-être il n'a été fait appel à leur générosité pour une cause qui en fût plus digne ${ }^{48} \gg$. Le résultat sera à la hauteur de l'appel : "Je ne crois pas que, durant mon administration du moins, une quête diocésaine ait jamais produit un aussi beau résultat ${ }^{49}$ ».

La quête pour la reconstruction de la Basilique de Sainte-Anne de Beaupré représente certainement le plus grand effort jamais déployé par $\mathbf{M}^{\mathrm{gr}}$ LaRocque et son clergé en faveur d'une quête commandée. En plus de faire lire en chaire la lettre pastorale collective rédigée par le cardinal Bégin, l'évêque de Sherbrooke envoie aux curés des recommandations supplémentaires :

Que d'ici au jour de la collecte, vous intéressiez vos fidèles à cette œuvre, que vous leur en fassiez comprendre toute la

46 Circulaire du 7 mai 1900 (incendie de Hull), in Mand. Sherbrooke, t. IV, p. 383. Voir aussi t. VI, p. 6.

47 Lettre de W.-A. Mackenzie, délégué du cardinal Bourne, reproduite in Mand. Sherbrooke, t. VIII, pp. 160-164.

48 Circulaire du 19 février 1916, in Mand. Sherbrooke, t. VII, pp. 408409.

49 Mand. Sherbrooke, t. VII, p. 423. 
nécessité et l'urgence, et le résultat, nous aurons la consolation de le constater, dépassera les espérances que nous avons de recueillir une offrande de $\$ 1.00$ par famille, en moyenne. Il y a, sans doute, dans toutes les paroisses, des familles pauvres qui ne peuvent pas faire cette offrande; mais, grâce au zèle que vous mettrez pour assurer le succès de cette quête, il n'y a pas de doute que les familles plus à l'aise compenseront, en donnant $\$ 2.00, \$ 5,00, \$ 10.00$ et même davantage; parce que, le pouvant, elles tiendront à donner à la Bonne Sainte Anne ce témoignage de leur gratitude pour les faveurs déjà reçues ou pour s'en assurer de nouvelles qu'elles désirent obtenir ${ }^{50}$.

On pourrait croire, cependant, que le succès de ces quêtes 《 uniques » provient autant de leur objet que des recommandations de l'évêque. Sans nier ce fait, nous pourrons constater l'impact des exhortations épiscopales de manière beaucoup plus directe en examinant le résultat des nouvelles quêtes annuelles instituées dans le diocèse. Considérons six de ces nouvelles quêtes et voyons la courbe qu'elles suivent durant les premières années où elles sont faites (Graphique VI).

Les conclusions sont évidentes : malgré la croissance régulière du produit de l'ensemble des quêtes, les nouvelles quêtes subissent toutes une diminution après la première année. Une analyse plus poussée montre que le phénomène exceptionnel n'est pas tant la diminution de la deuxième année que la poussée de la première. En effet, la plupart de ces nouvelles quêtes en remplacent d'autres, et il y a un bond considérable entre le produit de l'ancienne quête et celui de la nouvelle. Le meilleur exemple est celui de l'Euvre des Vocations Sacerdotales. La quête qui est remplacée, celle des Ruthènes, produisait quelque $\$ 400$. par année; la nouvelle quête rapporte en 1920 un montant de plus de $\$ 2000$., qui se stabilisera les années suivantes aux environs de $\$ 1300$.

Or ces quêtes nouvelles sont toutes annoncées et recommandées par l'évêque. Il est tout naturel que l'impact de ces circulaires spéciales se fasse surtout sentir la première année, et on peut, en quelque sorte, le mesurer par l'écart qui sépare les deux premières années.

50 Circulaire du 24 juillet 1922, in Mand. Sherbrooke, t. VIII, pp. 223224. 


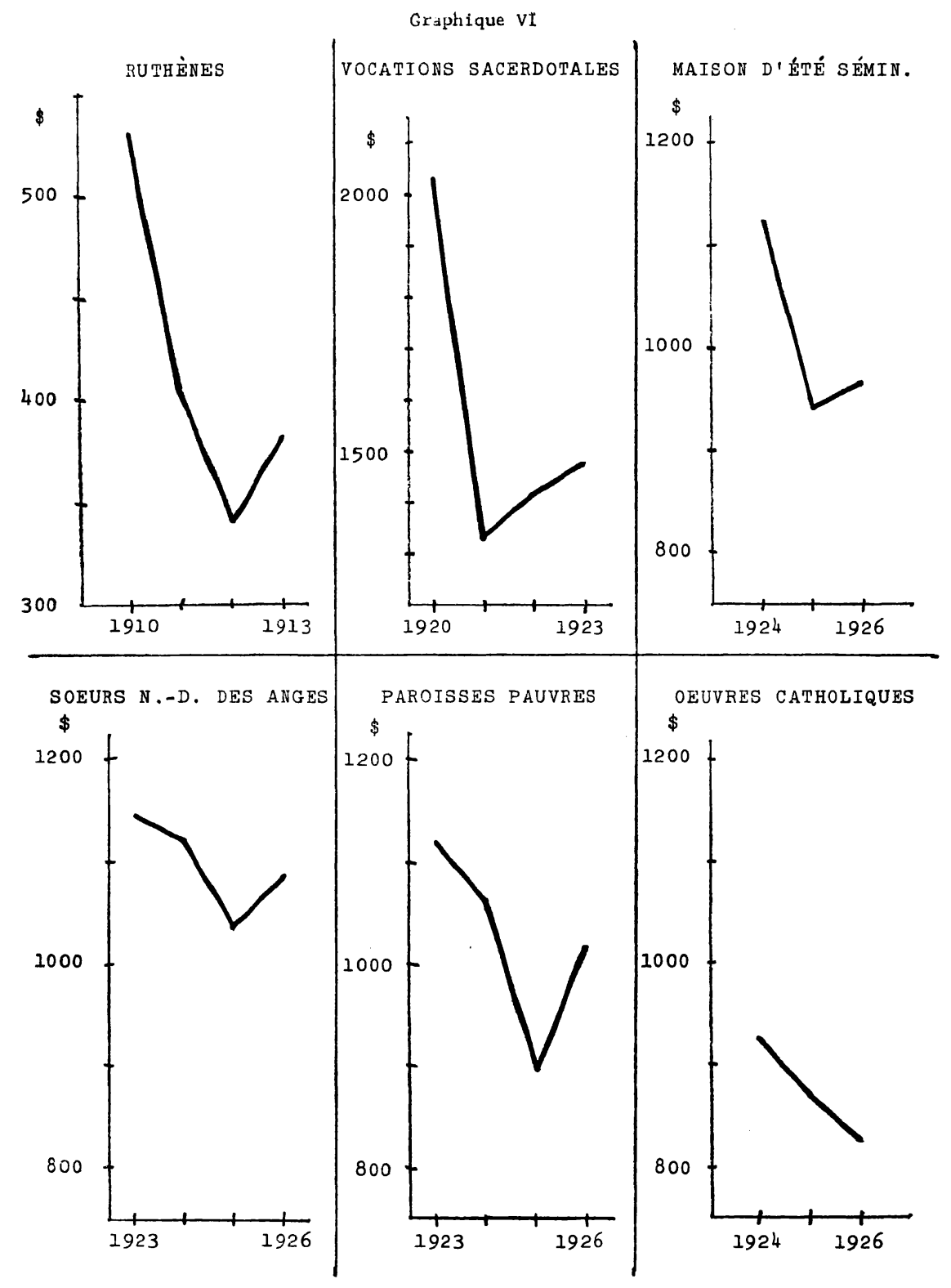

Si l'action de l'évêque est importante, celle de son clergé ne l'est pas moins. Il faudrait, pour bien la mesurer, dépouiller des Cahiers de prônes et voir comment les quêtes commandées étaient effectivement annoncées. Dans ses exhortations, $\mathbf{M}^{\mathrm{gr}}$ LaRocque relie 
d'ailleurs l'administration ponctuelle des quêtes ordonnées au zèle sacerdotal :

N'est-il pas évident que ces œuvres diocésaines dont la prospérité dépend surtout du soin que le prêtre met à les cultiver et promouvoir, ont un rapport direct avec ce qui constitue l'objet même du zèle sacerdotal ? 51

L'évêque termine la même circulaire par cette exhortation, bien caractéristique de la mentalité de l'époque :

Ne craignez done jamais de fatiguer, d'importuner vos ouailles par ces appels souvent répétés à leur générosité et à leur esprit de foi. Au soin, à la chaleur avec lesquels vous solliciterez leurs aumônes, les fidèles sentiront que vous avez vraiment à cœur le succès des œuvres que vous leur recommandez; ils en seront édifiés et touchés. Subissant l'heureuse contagion de votre zèle, ils n'hésiteront pas à faire les quelques petits sacrifices d'argent que vous leur demanderez. Ils donneront avec bonheur et s'attireront ainsi les bénédictions de Dieu : hilarem enim datorem diligit Deus (2 Cor., IX, 7) ${ }^{52}$.

Un autre phénomène à souligner à propos de ces quêtes est celui de la rivalité entre les paroisses. En présentant chaque année le Rapport des Euvres diocésaines, l'évêque invite souvent les curés à ce genre d'émulation :

Par des rapprochements et des comparaisons édifiantes, chacun pourrait ainsi se rendre compte lui-même si vraiment la paroisse dont il a charge, occupe, dans l'ordre de la charité, le rang d'honneur qui lui revient ${ }^{53}$.

En 1923, on changera même le mode de présentation du Rapport, pour faciliter de telles comparaisons. $\mathbf{M}^{\text {gr }}$ LaRocque s'en explique :

Croyant qu'il faut rendre à chacun selon ses œuvres et donner un honneur plus grand à qui le mérite davantage, j'ai fait disposer les noms des paroisses, non plus par ordre alphabétique, mais par ordre de générosité dans l'aumône. En même temps que cette disposition facilite, me semble-t-il, l'examen de conscience de chaque paroisse pour savoir si elle est au rang d'honneur qu'elle doit occuper dans sa coopération matérielle aux bonnes œuvres, elle sera une pieuse émulation de

51 Circulaire du 24 février 1902, in Mand. Sherbrooke, t. V, p. 66.

52 Ibid.

53 Circulaire du 9 juin 1919, in Mand. Sherbrooke, t. VII, p. 555. 
générosité qui conservera au diocèse de Sherbrooke sa belle réputation, si justement acquise, de diocèse progressif dont les œuvres fleurissent et se développent, grâce à la piété, à l'inlassable générosité des fidèles et des pasteurs qui, sous l'inspiration de leur foi, comprennent toute la grandeur et les mérites de l'aumône et la font largement 54 .

Il resterait sans doute bien d'autres facteurs à examiner pour expliquer dans le détail les variations du produit des quêtes, en particulier du côté de la population catholique elle-même. À mesure que nous connaîtrons mieux les conditions économiques et sociales, de même que les attitudes mentales des habitants des Cantons de l'Est et du Québec, nous pourrons mieux apprécier leur contribution aux œuvres de l'Église.

Notre étude pourrait se prolonger dans deux nouvelles directions. Il y aurait d'abord une analyse à faire au niveau des paroisses. D'autre part, il est possible d'étendre la recherche à d'autres diocèses et de comparer les résultats de différents diocèses entre eux. Les sondages que nous avons faits montrent qu'il existe des Rapports des auvres dans d'autres diocèses et que plusieurs des quêtes commandées se retrouvent partout ${ }^{55}$.

L'étude des quêtes commandées - et d'une manière plus large, de l'ensemble des auvres d'un diocèse - peut apporter beaucoup si, à côté des chiffres et des courbes rigoureusement analysés, on peut arriver à atteindre le niveau des attitudes et des mentalités. Au-delà du produit des quêtes, il reste à découvrir le geste, parfois routinier ou ostentatoire, parfois aussi sensible ou fervent, de celui et de celle qui déposent leur offrande dans le plateau.

GuY LAPERRIÈRE,

Département d'Histoire, Université de Sherbrooke.

54 Circulaire du 1er février 1923, in Mand. Sherbrooke, t. VIII, pp. 285286.

55 Nous avons fait des sondages dans les Mandements des diocèses de Québec, Montréal et Saint-Hyacinthe, pour les années 1900 et 1920 . Signalons qu'à Montréal, en 1920, le compte rendu est extrêmement détaillé pour l'Æuvre du Denier de Saint-Pierre (Mand. Montréal, t. XVI, pp. 312-345). 